\title{
Competition and bistability of longitudinal modes in a Raman laser
}

\author{
R. Corbalán, ${ }^{1}$ J. Cortit, ${ }^{1}$ and F. Prati ${ }^{2}$ \\ ${ }^{1}$ Universitat Autònoma de Barcelona, 08193 Bellaterra, Spain \\ ${ }^{2}$ Università degli studi di Milano, via Celoria 16, 20133 Milano, Italy
}

(Received 27 March 1995)

\begin{abstract}
A model for Raman lasers including several longitudinal modes is analyzed. Depending on the choice of the parameters the system can exhibit single-mode emission, wide bistability domains, and self-pulsing. The latter is often characterized by two frequencies, which are clearly related to single-mode and multimode instabilities, in agreement with the interpretation of earlier experimental results.
\end{abstract}

PACS number(s): 42.55.Ye, 42.65.Pc

The Raman laser is an optically pumped three-level laser in which both the pump and the laser field (frequencies $\omega_{P}$ and $\omega_{L}$, respectively) are far off resonance (see Fig. 1). This system is commonly used to produce high gain steady-state lasing emissions in the mid- to far infrared spectral region [1]. In addition, this system can show a very rich dynamical behavior [2], including single-mode pulsations and routes to chaos characterized by period doubling and intermittency [3]. The single-mode plane-wave model of the Raman laser has been already studied in great detail, and experimentally accessible parameters for which laser emission can be periodic or chaotic have been found [4-6].

Yet an important experimental evidence, namely, multimode emission, has not been taken into account in previous theoretical analyses. The single-mode model predicts that unstable behavior is associated with large cavity detuning. But as the cavity detuning becomes comparable with the free spectral range of the cavity, another longitudinal mode is likely to be excited. Therefore, the effects of single-mode and multimode instability should be visible under the same parametric conditions. The observation of two dynamical frequencies reported in $[7,8]$ confirms this picture.

We start from the standard set of equations for the singlemode Raman laser [6] written in a notation such that all variables and parameters are dimensionless:

$$
\begin{gathered}
\frac{d F}{d t}=-k\left\{\left(1-i \frac{\delta \Omega}{k}\right) F-2 C\left[\frac{\gamma}{4} P+i \frac{F(D-1)}{2 \delta}\right]\right\}, \\
\frac{d P}{d t}=\frac{4 \beta^{2}}{\gamma \delta^{2}} F D-(1+i \Delta) P \\
\frac{d D}{d t}=-\gamma\left[\frac{1}{2}\left(F^{*} P+F P^{*}\right)+D-1\right]
\end{gathered}
$$

where

$$
\Delta=\delta_{R C}-\delta \Omega+\left(\beta^{2}-|F|^{2}\right) / \delta .
$$

We have introduced some changes of variables with respect to [6] in order to make our equations more similar to the Maxwell-Bloch equations commonly used to describe a twolevel laser: the variables $\Delta, W$, and $P$ used in [6] are in our notation $-\Delta,-D$, and $-\gamma \delta P /(4 \beta)$, respectively. The dimen- sionless time variable in Eqs. (1) is scaled to $\gamma_{\perp}$, the relaxation rate of $P$, and therefore all frequencies are given in units of $\gamma_{\perp}$.

With our notations $\beta$ and $F$ are equal to half the Rabi frequencies associated with the pumping and lasing transitions, respectively. $P$ and $D$ denote as usual atomic coherence and difference of population, respectively, of the effective two-level system formed by the levels 2 and 1 .

The three-level scheme is shown in Fig. 1: $\omega_{02}$ and $\omega_{01}$ are the frequencies of the transitions $2 \leftrightarrow 0$ and $1 \leftrightarrow 0$ and $\delta$ is the detuning between the frequency $\omega_{P}$ of the pump beam and the atomic resonance $\omega_{02}$. We define the Raman frequency $\omega_{R}=\omega_{01}+\delta$. The actual laser frequency $\omega_{L}$ coincides with $\omega_{R}$ only when it is resonant with some cavity frequency $\omega_{C}$. In general, one has $\omega_{L}=\omega_{R}+\delta_{R}$. It is useful to write $\delta_{R}=\delta \Omega-\delta_{R C}$, where $\delta \Omega=\omega_{L}-\omega_{C}$ and $\delta_{R C}=\omega_{R}-\omega_{C}$. The reason for splitting the detuning $\delta_{R}$ in the two terms $\delta \Omega$ and $\delta_{R C}$ is that, while $\delta_{R C}$ is one of the parameters of the system, $\delta \Omega$ is a function of all the parameters of the system, including $\delta_{R C}$. Finally, $k$ is the cavity linewidth, $\gamma$ is the decay rate of the difference of population $D$, and $2 C$ is a cooperativity parameter proportional to the density of atoms.

As already discussed in Ref. [6], Eqs. (1) are a good approximation to the system of equations that describe an op-

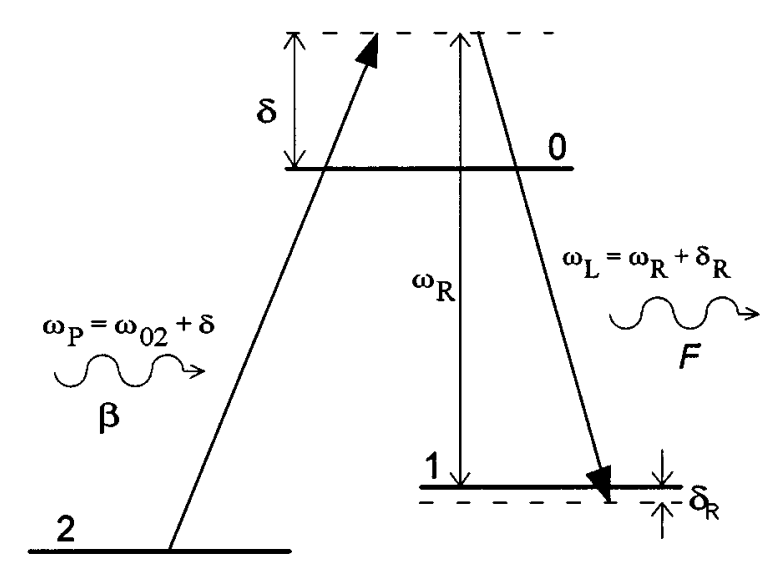

FIG. 1. Scheme of the Raman system considered in this paper. $\omega_{P}$ is the frequency of the pump beam. $\omega_{L}$ is the frequency of the generated laser beam. $\delta_{R}$ is the detuning between the Raman frequency $\omega_{R}=\omega_{01}+\delta$ and the laser frequency $\omega_{L}$. 
tically pumped three-level laser [9] in the Raman limit, namely, $\delta \gg 1, \delta \Omega-\delta_{R C} \ll \delta$ and $|F|^{2}, \beta^{2} \ll \delta^{2}$. The first two inequalities establish the condition that the pump and laser beams are far off resonance from the $2 \leftrightarrow 0$ and $0 \leftrightarrow 1$ atomic transitions, respectively, and that, at the same time, a twophoton transition involving the absorption of one photon from the pump beam and the simultaneous emission of one photon to the generated laser beam is nearly resonant with the $2 \leftrightarrow 1$ atomic transition. The third inequality ensures that far-off-resonant conditions persist in the presence of broadening arising from Rabi splitting of the pump and lasing transitions. Under these conditions, the optical coherences between levels 0 and 2 and between levels 0 and 1 can be adiabatically eliminated and the population of the upper level 0 is practically unaffected by the pumping and laser fields and may be set to zero. As a consequence, the three-level system is described by only two variables: the two-photon atomic coherence $P$ and the population difference $D$ between levels 2 and 1 . The pump laser amplitude $\beta$ is assumed to be a space- and time-independent control parameter; the remaining evolution equation (1a) gives the amplitude $F$ of the generated laser.

We now abandon the single-mode approximation and assume that the electric field depends on the longitudinal coordinate $z$. When one takes into account the boundary conditions imposed by the mirrors of the resonator that contains the active medium [10], one finds that the slowly varying envelope $F(z, t)$ of the laser field obeys the equation

$$
\begin{aligned}
\frac{\partial F}{\partial t}+\frac{c L}{\gamma_{\perp} \mathscr{L}} \frac{\partial F}{\partial z}= & -k\left\{\left(1-i \frac{\delta \Omega}{k}\right) F\right. \\
& \left.-2 C\left[\frac{\gamma}{4} P+i \frac{F(D-1)}{2 \delta}\right]\right\},
\end{aligned}
$$

where $\mathscr{B}$ is the cavity round-trip and $L$ is the length of the active medium. The time variable is now defined in such a way that it includes the delay occurring in that part of the cavity not occupied by the active medium [10]. Therefore the boundary conditions for the electric field are isochronous

$$
F(0, t)=F(L, t)
$$

and $F$ can be expanded in Fourier components, or longitudinal modes, labeled by the index $n, n=0$ being the reference mode

$$
F(z, t)=\sum_{n=-\infty}^{+\infty} f_{n}(t) \exp \left(i k_{n} z\right), \quad k_{n}=\frac{2 \pi}{L} n
$$

We call $\omega_{n}$ the frequency of the $n$th mode. The frequency separation between two consecutive modes is the normalized free spectral range $\alpha=2 \pi c / \mathscr{L} \gamma_{\perp}$. We take as reference cavity frequency the frequency of mode $0: \omega_{C}=\omega_{0}$.

By projecting Eq. (3) onto each mode, we obtain an infinite set of dynamical equations for each mode amplitude $f_{n}$

$$
\begin{aligned}
\frac{d f_{n}}{d t}= & -i n \alpha f_{n}-k\left\{\left(1-i \frac{\delta \Omega}{k}\right) f_{n}-2 C \int_{0}^{2 \pi} \frac{d \theta}{2 \pi} e^{-i n \theta}\right. \\
& \left.\times\left[\frac{\gamma}{4} P+i \frac{F(D-1)}{2 \delta}\right]\right\}
\end{aligned}
$$

where we have introduced the dimensionless angular variable $\theta=k_{1} z$. Integration over $\theta$ by $2 \pi$ is equivalent to integration over $z$ along all the atomic medium of length $L$.

Since we limit the mode expansion to the electric field, Eqs. (1b) and (1c) remain unchanged, apart from the fact that now $F, P$, and $D$ are functions of time and of $\theta$. Equations (6), (1b), and (1c) admit an infinite number of single-mode stationary solutions $F^{\text {st }}=f_{n}^{\text {st }} e^{i n \theta}$ corresponding to each mode $n$.

The single-mode stationary solution $n$ can be destabilized through two different mechanisms: (a) amplification of any fluctuation of mode $n$ itself (single-mode instability) and (b) amplification of any fluctuation of the couple of modes $m$ and $2 n-m$, with $m \neq n$ (multimode instability).

We assume that the Raman resonance $\omega_{R}$ always lies between $\omega_{0}$ and $\omega_{1}$. Therefore, the stationary solutions with lowest threshold are either 0 or 1 and we limited our study to them. However, in the linear stability analysis other modes come into play: for instance, mode 0 is destabilized at the same time by modes 1 and -1 and mode 1 by modes 0 and 2 . In our numerical simulations we always considered seven modes, from -3 to 3 , always checking that the intensities of modes \pm 3 were some orders of magnitude smaller than the others. Sometimes we have also run the program including other modes, up to 15 , and we have verified that their intensity is always very small. We can then conclude that, with our choice of parameters, the laser behaves essentially as a two-mode laser, in the sense that modes different from 0 or 1 are never selected by the laser as stationary solutions and their intensities in all dynamical regimes are always very small. That is the reason why in the figures we show only the intensities of modes 0 and 1 .

The choice of the right parameters is a delicate problem in the study of the Raman laser because of their large number and because some of them can hardly be estimated with sufficient accuracy in the experiments. Thus we decided to adopt exactly the same parameters used in [4], namely, $k=3$, $2 C=60, \gamma=0.83$, and $\delta=14$. In addition, we took the value $\alpha=8$ for the free spectral range, on the basis of the data reported in [3]. The free parameters are the pump amplitude $\beta$ and the cavity detuning $\delta_{R C}$.

In Fig. 2 we plotted the lasing thresholds and instability boundaries, calculated using a standard linear stability analysis, for the two single-mode solutions. The value of the detuning $\delta_{R C}$ varies from 0 (perfect resonance with mode 0 ) to $8\left(\delta_{R C}=\alpha\right.$, perfect resonance with mode 1$)$. This means that the atomic line is always between the two modes. In this picture the label $N a M$ denotes the instability boundary of mode $N$ against mode $M$ and mode $2 N-M$. Mode 0 is unstable to the right of line $0 \mathrm{a} 0$ due to a single-mode instability and above line 0a1 due to a multimode instability caused by modes 1 and -1 . Mode 1 is unstable below line $1 \mathrm{a} 0$ due to a multimode instability caused by modes 0 and 2 and below line $1 \mathrm{a}-1$ due to a multimode instability caused by modes -1 and 3 .

It must be kept in mind that in a single-mode Raman laser the first bifurcation (threshold bifurcation) may be either super- or subcritical, depending on the values of the parameters. The first bifurcation is subcritical when $\delta_{R C}$ is larger than a certain critical value $\delta_{R C}^{c}$ given by 


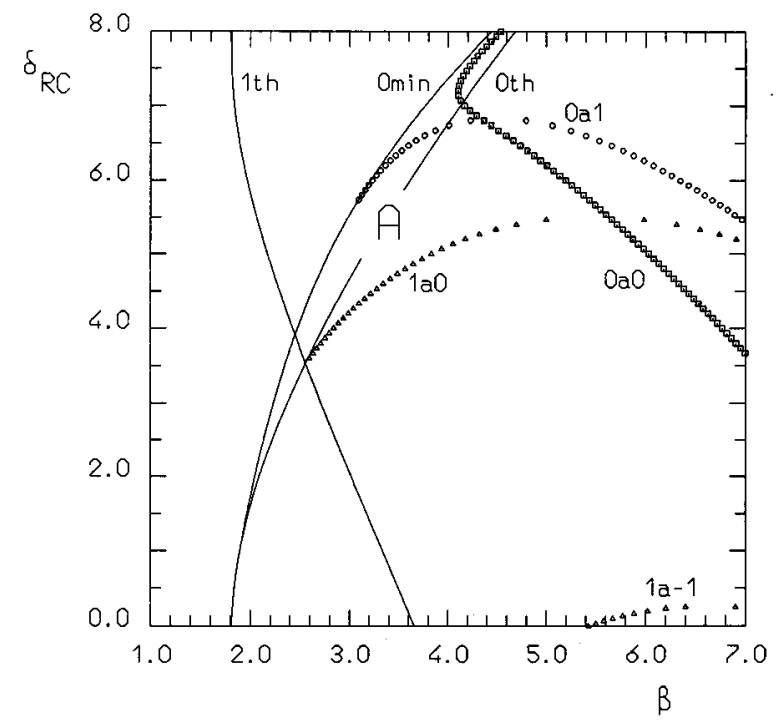

FIG. 2. Stability domains of the two single-mode solutions 0 and 1 . Mode 0 is unstable to the right of line $0 \mathrm{a} 0$ due to a singlemode instability and above line $0 \mathrm{a} 1$ due to a multimode instability. Mode 1 is unstable below lines $1 \mathrm{a} 0$ and $1 \mathrm{a}-1$ due to multimode instabilities. 0th and 1th are the threshold lines for modes 0 and 1, respectively. Since the threshold bifurcation of mode 0 is subcritical for some values of $\delta_{R C}$, the single-mode solution 0 also exists below threshold inside the region delimited by lines 0th and 0min. In region $A$ both single-mode solutions 0 and 1 are stable.

$$
\begin{aligned}
\delta_{R C}^{c}= & \frac{1}{8} \frac{2 C}{\delta}\left[2(\gamma+2 k)-\left(\frac{\gamma+2 k}{1+k}\right)^{2}\right] \\
& \times\left\{1-\left[1-16\left(\frac{\delta}{2 C}\right)^{2}\left(\frac{1+k}{\gamma+2 k}\right)^{2}\right]^{1 / 2}\right\} .
\end{aligned}
$$

If the argument of the square root is negative, the expression of $\delta_{R C}^{c}$ is useless and the first bifurcation is supercritical for all values of $\delta_{R C}$. If the argument of the square root is positive, $\delta_{R C}^{c}$ is positive since $\gamma$ is always smaller than 1 . The previous discussion can be summarized easily in the following way: the first bifurcation is supercritical if the atomic line is on the left of the cavity line ( $\delta_{R C}$ negative) and it becomes subcritical when the atomic line is sufficiently displaced to the right of the cavity line. Therefore, in our case the bifurcation is always supercritical for mode 1 and can be subcritical for mode 0 . That is why in Fig. 2 we drew just one threshold line (1th) for mode 1 and two lines for mode 0: 0th is the lasing threshold and $0 \mathrm{~min}$ is the minimum value of $\beta$ for which the stationary solution exists; it is smaller than threshold when the first bifurcation is subcritical.

Two regions in Fig. 2 are of particular interest: region $A$, in which both solutions are stable, and the region to the right of line $0 \mathrm{a} 0$, where solution 0 is always unstable. Let us consider first region $A$. Two kinds of bistable behaviors are possible: either varying the detuning $\delta_{R C}$ or varying the pump amplitude $\beta$. In order to show the first kind of bistability, we kept the pump amplitude fixed at $\beta=4$ and we let the detuning $\delta_{R C}$ vary from 4.8 to 7 (more than one-fourth of the free spectral range) and back. This can be accomplished in a real experiment by means of a piezoelectric translator (PZT). A large hysteresis cycle between modes 0 and 1 is visible in

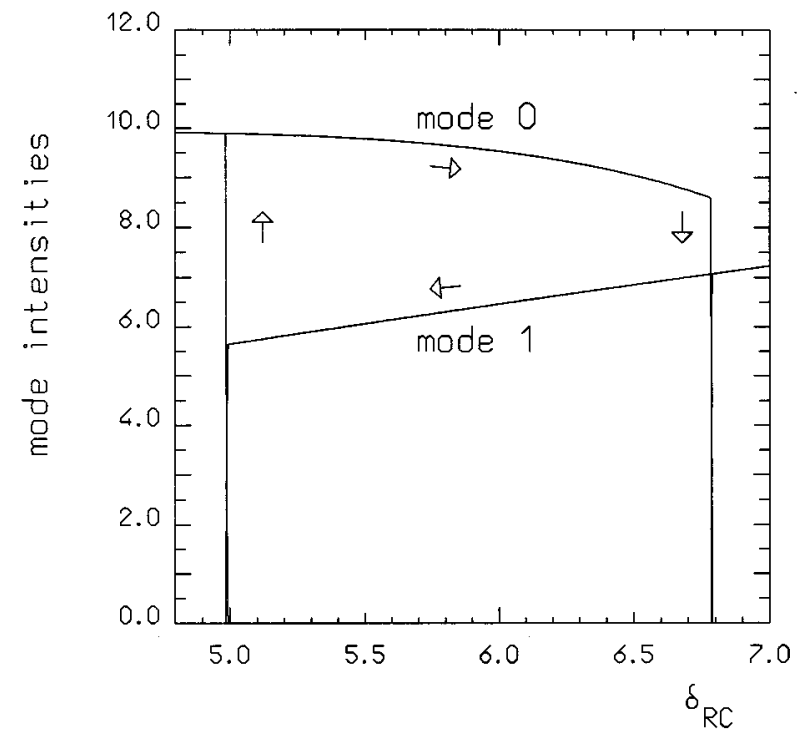

FIG. 3. Adiabatic sweep (forward and backward) of the detuning $\delta_{R C}$ for $\beta=4$. A large hysteresis cycle is visible.

Fig. 3. In the forward sweep the laser switches to mode 1 at $\delta_{R C} \simeq 6.78$; the reverse switch occurs in the backward sweep at $\delta_{R C} \simeq 4.98$. It must be noted that the upper and lower branches in the bistable domain differ not only for the output intensity (as in standard optical bistability) but also for the frequency. The heterodyne power spectra obtained by making the laser output in the upper and lower branches at $\delta_{R C}=6$ interfere with a probe beam whose frequency coincides with the empty cavity frequency of mode 0 gives the following results: the beat frequency is 6.09 for mode 0 and 7.87 for mode 1 , resulting in a frequency difference of 1.78 between the two single-mode solutions, not far from the mode-pulled free spectral range $\alpha /(1+k)=2$.

Bistability between the nonlasing and lasing states has been already reported for a single-mode Raman laser [4], but in a two-mode laser the bistability domain can be much more extended. Bistability between longitudinal modes was found also in two-level lasers [10], but in Raman lasers it is greatly enhanced by the asymmetric shape of the gain curve [4]. Moreover, in Raman lasers bistability with pump amplitude as control parameter is possible too.

To show this we fixed $\delta_{R C}=5$ and let $\beta$ vary from 2 to 4.2 and back. The hysteresis cycle is shown in Fig. 4, where we have superimposed on the results of dynamical simulations (solid lines) the two steady-state curves for modes 0 and 1 (dashed lines). It is evident that in this case bistability stems from the different kind of lasing bifurcation for the two modes. For mode 1 the bifurcation is supercritical and the threshold value of $\beta$ is smaller than for mode 0 , which, on the contrary, bifurcates subcritically. Thus, by increasing $\beta$, the laser first follows the steady-state curve of mode 1, until it becomes unstable at $\beta \simeq 3.96$, and then jumps discontinuously to mode 0 , whose intensity is larger. By decreasing $\beta$, the laser always remains on mode 0 up to the turning point of the steady-state curve, where it precipitates again on mode 1.

The phenomena of bistability typical of region $A$ is related essentially to multimode instability: one mode is re- 


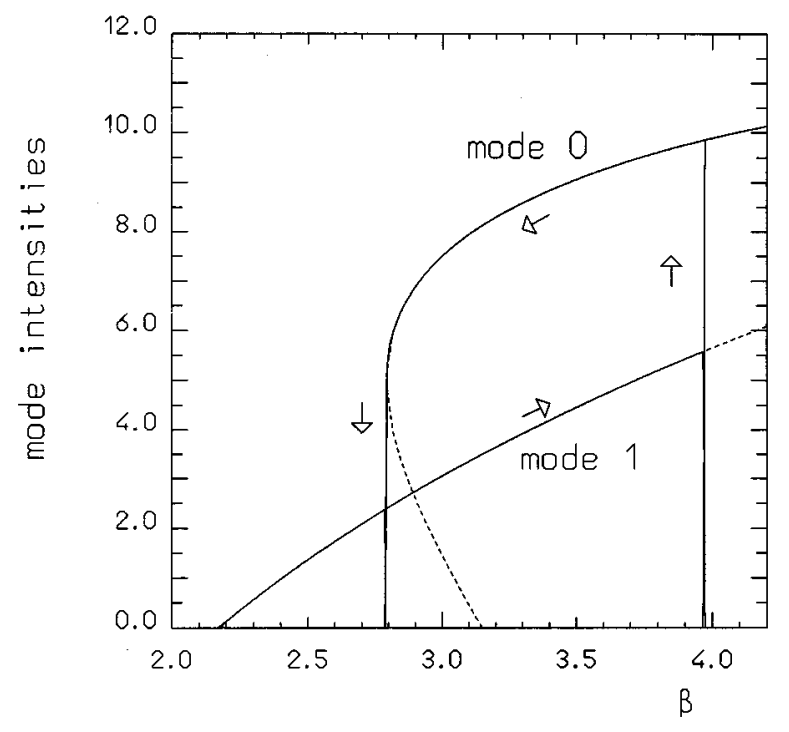

FIG. 4. Adiabatic sweep (forward and backward) of the pump amplitude $\beta$ for $\delta_{R C}=5$. The solid lines show the dynamical results and the dashed lines the steady-state curves for mode 0 and mode 1 .

placed by the other when it becomes unstable with respect to it. There is no self-pulsing associated with this kind of instability.

On the contrary, in the region to the right of line $0 \mathrm{a} 0$ mode 0 is always unstable because of single-mode instability, which leads to self-pulsing, and this instability can coexist with a multimode instability, giving rise to interesting dynamical phenomena. To investigate them we performed a dynamical sweep of $\delta_{R C}$ from 5.5 to 7 , with $\beta=5.4$. The evolution of mode intensities is shown in Fig. 5, where four distinct regions are visible. In region 1 we are below line $0 \mathrm{a} 0$ of Fig. 2 and the laser emits stably mode 0 . Crossing line $0 \mathrm{a} 0$ we enter region 2 , where undamped oscillations of mode 0

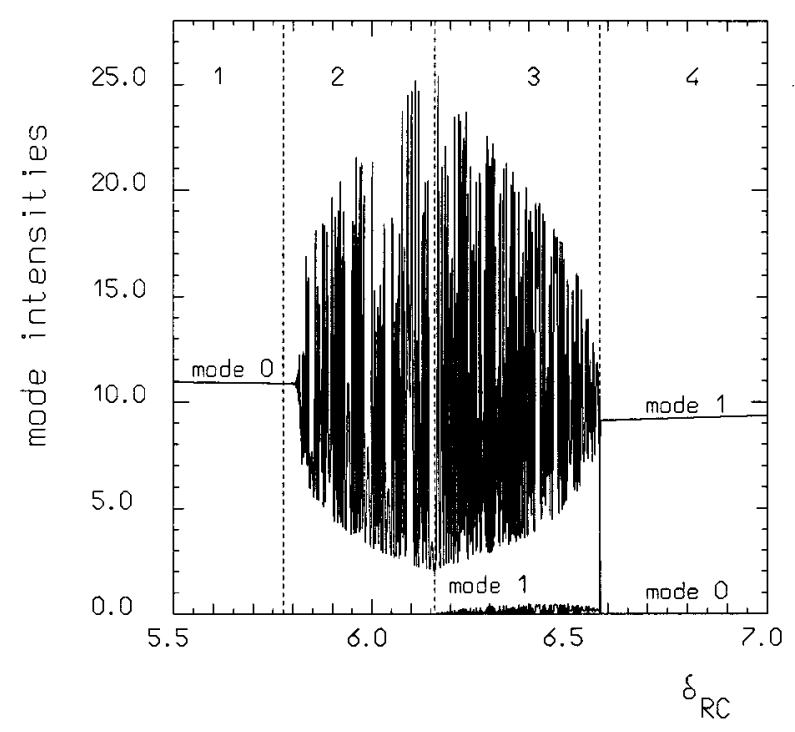

FIG. 5. Adiabatic sweep of the detuning $\delta_{R C}$ from 5.5 to 7 (forward) for $\beta=5.4$. The meaning of the four regions is explained in the text.
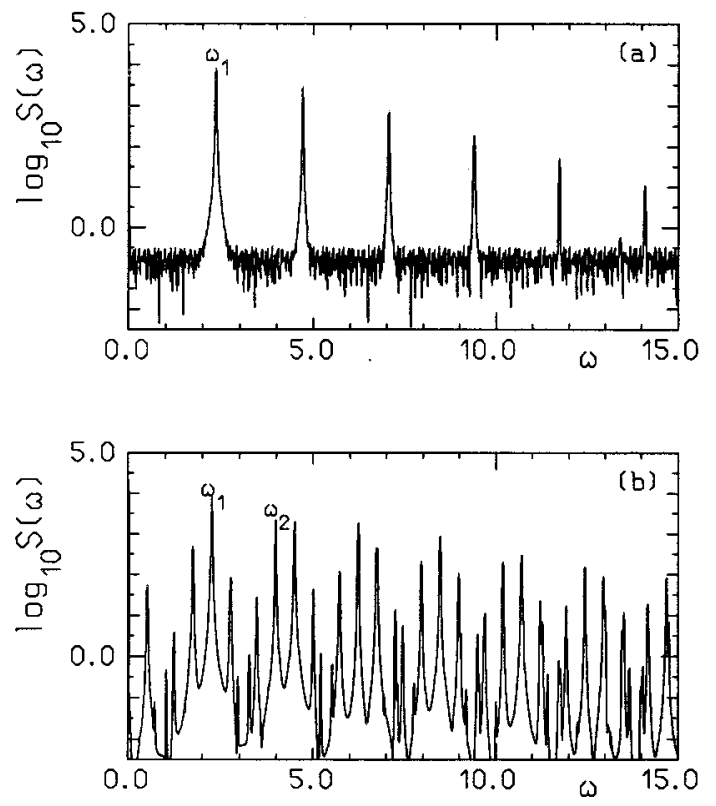

FIG. 6. Power spectra of the laser output for two different values of $\delta_{R C}$ belonging to regions 2 (a) and 3 (b) of Fig. 5 .

appear; since oscillations are due to single-mode instability, mode 1 is still off. Region 3 begins at about $\delta_{R C}=6.16$, when mode 1 enters to play. In this region the two modes coexist but the intensity of mode 1 is very small. The two modes beat one with the other and in addition their intensities are modulated because of the single-mode instability of mode 0 . At about $\delta_{R C}=6.58$ the laser switches to mode 1 and oscillations disappear. We stress that this occurs before crossing line 0a1, which is placed at $\delta_{R C}=6.60$. This is not strange because line $0 \mathrm{a} 1$ refers to the stability of the stationary solution 0 against mode 1 and it tells nothing about the stability of the dynamical solutions formed by the two modes 0 and 1 .

The power spectra for two values of $\delta_{R C}$ belonging to regions 2 and 3 are shown in Fig. 6. At $\delta_{R C}=6$ (region 2) the frequency $\omega_{1} \simeq 2.36$ due to single-mode instability is visible together with many harmonics. The power spectrum at $\delta_{R C}=6.4$ (region 3) is much more complex because, besides the frequency $\omega_{1}=2.26$ and its harmonics, the beat frequency $\omega_{2}=3.98$ and its multiples due to beating among different modes are present (beating between modes $N$ and $M$ gives a peak at $\left.|N-M| \omega_{2}\right)$. Moreover, the combinations of these two sets of frequencies are also visible. For instance, the first peak is the beat note $2 \omega_{1}-\omega_{2}$; the peaks to the left and to the right of $\omega_{1}$ are, respectively, $\omega_{2}-\omega_{1}$ and $3 \omega_{1}-\omega_{2}$; the peak to the left of $\omega_{2}$ is $2 \omega_{2}-2 \omega_{1}$. The beat frequency $\omega_{2}$ is always much larger than the one predicted by the mode pulling formula $\alpha /(1+k)=2$, which means that the two-mode solution is characterized by a strong nonlinear mode pushing.

In conclusion, we have demonstrated that under general conditions the dynamics of a Raman laser is dominated by the two modes that are closer to the Raman resonance. The parameter space displays large domains of bistability as well as domains characterized by the simultaneous presence of two dynamical frequencies, as observed in the experiments by Harrison and Biswas [7]. In the future we plan to improve 
our model by taking into account the Gaussian profile of pump and laser fields. The nonuniformity of the fields in the transverse plane is particularly important in a Raman laser because the atomic detuning depends on field intensity. Thus atoms placed at different points of the transverse plane experience different atomic detunings.
We acknowledge useful discussions with M. Brambilla. J.C. acknowledges the DGU (Generalitat de Catalunya) for a grant (Grant No. FI/91-186). F.P. acknowledges the CESCA (Centre de Supercomputació de Catalunya) for a grant. Financial support from the DGICYT (Project No. PB92-0600$01)$ is also acknowledged.
[1] N. G. Douglas, Millimetre and Submillimetre Wavelength Lasers (Springer, Berlin, 1989).

[2] R. G. Harrison and I. A. Al-Saidi, Opt. Commun. 54, 107 (1985)

[3] D. J. Biswas and R. G. Harrison, Opt. Commun. 54, 112 (1985).

[4] R. G. Harrison, Weiping Lu, and P. K. Gupta, Phys. Rev. Lett. 63, 1372 (1989).

[5] Weiping Lu and R. G. Harrison, Phys. Rev. A 41, 6563 (1990).
[6] Weiping Lu and Robert G. Harrison, Phys. Rev. A 43, 6358 (1991).

[7] R. G. Harrison and D. J. Biswas, Phys. Rev. Lett. 55, 63 (1985).

[8] R. G. Harrison, I. A. Al-Saidi, and D. J. Biswas, IEEE J. Quantum Electron. QE-21, 1491 (1985).

[9] M. A. Dupertuis, R. R. E. Salomaa, and M. Siegrist, Opt. Commun. 55, 370 (1985).

[10] L. M. Narducci, J. R. Tredicce, L. A. Lugiato, N. B. Abraham, and D. K. Bandy, Phys. Rev. A 33, 1842 (1986). 